monitoring. A literature review indicates that previous clinical trials have failed to demonstrate statistically significant occurrence of serious haematological abnormalities. Our experience shows that neutropenia can indeed be a serious side effect. In both cases, prompt reversal of neutropenia was observed upon discontinuation. Given the cost and discomfort associated with routine investigations, we recommend large multicentric observation studies with the aim of creating a unified standard.

\section{CHILDHOOD MENINGITIS (EXPERIENCE OF THE PEDIATRIC DEPARTMENT FOR A YEAR)}

doi:10.1136/archdischild-2012-302724.0855

ND Righi, S Brahmi, H Zerguine, S Tebbal. CHU Benflis Touhami, Universite de Batna, Park Aforage, Algeria

Introduction Meningitis is one of concern in Pediatric Infectious Diseases icts with impact, the Epidemiological Profile, ict severity in the short and long term Especially if it is Caused by bacteria, and Especially the interest of her proper care.

Objective Evaluate the epidemiology, bacteriology, etiology of meningitis and How They support and Their Future.

Material and Methods A retrospective study of records of patients hospitalized in the Pediatric University Hospital DURING 2010 BATNA. 70 Patients Were the subject of this study.

Results Both sexes are Affected with a male predom inance. The Age Group Most Affected is 30 days to 05 years $55.71 \%$ (range of 30 daysand 15 years).

A marked Increase in incidence Between April and August (61 boxes).

Fever WAS Noted in $100 \%$ of our patients, 32 patients complained of headache. The neck stiffness WAS Noted in 13 patients, 02 patients presented seizures.

Cytological CSF study objectified That HAS $57 \%$ of patients Had a number of elements Between 10 and 500 cells $/ \mathrm{mm} 3$ with presence of neutrophils and lymphocytes in $40.4 \%$.

The hypoglycorrhachia WAS present in $55.7 \%$ of cells, the hyperalbuminorrachie in $31.4 \%$ of boxes. Leukocytosis WAS present in $63.33 \%$ cells, leukopenia in boxes $26.66 \%$.

67\% Of Our Patients Had a positive CRP, 61.8\% Had an ESR.

Conclusion Confirmed this study the frequency of meningitis in children. The preponderance of the viral origin Which corresponds to literature.

\section{SALMONELLA ENTERITIDIS MENINGITIS IN A CASE REPORT}

doi:10.1136/archdischild-2012-302724.0856

ND Righi, S Brahmi, H Zerguine, T Bendhib, A Kasseh Laàouar. CHU Benflis Touhami, Universite de Batna, Park Aforage, Algeria

Introduction Salmonella are the usual agents of gastrointestinal infections caused by ingesting food or water contaminated responsible for gastroenteritis, infectious forms with predominant symptoms represent $5-10 \%$ of all salmonellosis They occur most often in patients malnourished, immunocompromised or sickle cell.

We report a case of salmonella meningitis complicated by pericarditis and septic shock.

Observation KM infants 3 months old born and residing in Patna, the third in a family of three EVBP, from a consanguineous marriage, was admitted at the pediatric ward on 10-12-2008 for: septic shock whose clinical examination objectified:

an altered state with general pallor CM, grunting, sclerema, bradypnea, tachycardia and mottled extremities neurological syndrome: hypertonia with generalized convulsion a PL which was performed: in favor of a purulent meningitis in Salmonella Enteritidis Inflammation testing strongly positive.
Echocardiography: pericardial electrophoresis HB: hemoglobin C. Conclusion The salmonella although they are responsible for gastroenteritis often with good prognosis under treatment in certain situations may give serious systemic infections and their prognosis remains reserved hence the interest to look at this type of infection an underlying pathological field.

Annuler les modifications.

Dictionnaire.

\section{EPIDEMIOLOGY OF BLOODY DIARRHOEA AMONG CHILDREN LESS THAN TEN YEARS OF AGE IN BAGHD}

doi:10.1136/archdischild-2012-302724.0857

WA-A0 Al-Kubaisy. Community Medicine, UiTM Faculty of Medicine, Shah Alam, Malaysia

Bloody diarrhea in young children is usually a sign of invasive enteric infection that carries a substantial risk of serious morbidity and death Among its important risk factors are poor environmental sanitation, unsafe water, poor personal hygiene \& artificial feeding In Iraq the sanitary condition \& general hygiene had been deteriorating during the last decade, particularly following the last war.

Objectives To identify prevalence \& risk factors associated withbloody diarrhea.

Methodology Cross - sectional study was conducted. Collecting 1500 children aging $<10$ years old, having diarrhea, from two pediatric teaching hospitals in Baghdad. Mothers Interviewing, clinical $\&$ stool laboratory examination were carried-out for each patients. Results Prevalence of bloody diarrhea was (28\%). No significant association between sex \& bloody diarrhea. Significantly, higher rate of bloody diarrhea among children; aging7-9 years $(66.6 \%)$ living in rural areas (41.9\%), their mothers were illiterates $(31.5 \%)$, household water from river (46.3\%) not using refrigerator for food storage $(34.1 \%)$, and children who were on exclusive bottle feeding $(36.5 \%)$. While insignificantly higher rate $(33.3 \%)$ among children of working mothers.

Entamoeba Histolytica was the main causative agents (83.58\%), with significant higher prevalence $(97.5 \%)$ among age group 1-3years. Prevalence of Non-typhoid salmonella or Shigella (4.28\%, $2.14 \%$ ) respectively. were found more among age $4-6$ years $(42.1 \%)$, (15.8\%) respectively.

Conclusion Bloody diarrhea highly prevalent in Baghdad (28\%). Entamoeba histolytica is the commonest causative agent Non breast fed baby at high risk. Therefore efforts should be directed toward encouraging exclusive breast feeding and, improvement of the basic infrastructures.

\section{ANALYSIS OF THE INCIDENCE OF GIARDIASIS IN CHILDREN WITH ATOPIC DERMATITIS}

doi:10.1136/archdischild-2012-302724.0858

E Adzhygafarova. Department of Propedeutics of Pediatrics, Crimea State Medical University named after S. I. Georgievsky, Simferopol, Ukraine

Atopic dermatitis holds the first place among children's allergic diseases. One of its proven ethiopathogenetic factors is parasitosis, affecting the course of dermatoses and leading to frequent recurrences and chronization of dermal processes.

Objective Studying the frequency of atopic dermatitis and giardiasis occurrence in children.

Materials and Methods In 2007-2011, 173 children aged 3-17 have been examined, who underwent treatment for atopic dermatitis in the Clinical Hospital of Simferopol. Their diagnosis were verified based on the patients' complaints, histories, clinical, biochemical and instrumental examinations.

Discussion Out of 173 examined children, 46 were diagnosed with giardiasis. Apart from typical symptoms of allergy, clinical findings 
included skin manifestations inherent to giardiasis: paleness, cheilitis, hyperkeratosis follicularis punctata and prolonged skin itching. As a result of reflex and toxicoallergic actions of Giardia, a syndrome of chronic endointoxication has prompted emergence of dyskinesia of the gallbladder and sphincter apparatus in $75 \%$ of patients with subsequent inflammation of the gastrointestinal tract (in $48 \%$ of patients). That further increased an antigenic load on their immune systems. SCORAD index in 22 infected children showed moderate severity of atopic dermatitis, whereas 18 patients proved to have a severe form.

Conclusion The analysis has shown that $27 \%$ of patients with atopic dermatitis were infected with giardiasis. These data require a use of a complex approach to the therapy of atopic dermatitis, more thorough examination of children for any pathology of the gastrointestinal tract, particularly, the examination of helminths.

\section{DERMATOLOGIC IMMUNE RECONSTITUTION INFLAMMATORY SYNDROME (IRIS) IN CHILDREN RECEIVING ART FROM A COMMUNITY OUTREACH PROGRAM IN KAMPALA}

doi:10.1136/archdischild-2012-302724.0859

'A Bagenda, 2J Mbabazi, ${ }^{3} \mathrm{M}$ Nalubega-Mboowa. 'International Health Sciences University, Entebbe; ${ }^{2}$ Department of Peadiatrics and Child Health, Mulago Hospital Kampala; ${ }^{3}$ Health Policy and Management, International Health Sciences University, Kampala, Uganda

Background Orikiriiza (2011) demonstrated that dermatological manifestations were the most common IRIS events in children receiving ART. We aimed to find the incidence of dermatologicalIRIS in children receiving ART for at least 12 weeks.

Methodology Retrospective review of medical charts for children who received ART between January 2010-December 2011. Nonadherent and regimen switch children excluded.

Results Total of 110 charts; median age 5.9 years (IOR 9.1months11.9years).70 females (63.6\%). 85children (77.2\%) baseline WHO stage III/IV. All children received septrin. Median time on ART was 24 weeks (IOR 13.2-40.8). Baseline CD4+\% was < 15\% for $77(70 \%)$, $>=15 \%$ for 33 children. Viral load $>399,000$ copies were 76 children $(69.1 \%)$ and $<=399,000$ for 34 children. PPE had the highest incidence (47cases) after ART initiation. 10cases of verrucae planae, 9 Kaposi Sarcoma, Herpes Zoster and Tinea corporis each, 8molluscum contagiosum, 4tinea capitis, 3HSV and 1varicella zoster. Median time to develop PPE was 3weeks (IOR 10.3-50. 1days). Increasing age associated with IRIS; highest between 5-12 years (age correlated with degree of immunosuppression).

Viral load after 3 months; < $1 \log 10$ decrease for 45 children $(40.9 \%),>=1 \log 10$ decrease for 65 children. Children who had $>=25 \mathrm{cells} / \mathrm{ul}$ change in CD4+\% (83 children) carried almost 3-fold risk for dermatologic -IRIS compared to children with $<25$ cells increase (69 Vs 9 cases [O.R 2.9 CI 1.40-11.02, p value 0.004]. No significant increased risk for dermatologic-IRIS based on viral load change.

Conclusion Prevalence of unmasking dermatological-IRIS was high. PPE accounted for highest mucocutaneous IRIS manifestations. Caregivers should be counseled about possible worsening of PPE with ART initiation.

\section{EPIDEMIOLOGICAL FACTORS AND FOOD: WHICH IS THE ROLE IN HELICOBACTER PYLORI RE-INFECTION IN PEDIATRIC AGE?}

doi:10.1136/archdischild-2012-302724.0860

T Sabbi. ASL RMG, Rome, Italy

Background Helicobacter pylori $(\mathrm{Hp})$ infection has been recognized as a cause of chronic gastritis, peptic ulcer, atrophic gastritis and gastric cancer. Its acquisition is related with poor socioeconomic conditions while the relationship of nutrition and $\mathrm{Hp}$ is still a question.

Aim To analyzed if socioeconomic factors and dietary contribute to Hp re-infection in pediatric age.

Patients and methods 150 patients (92 males; age range 5-16 years) with $\mathrm{Hp}$ infection treated and eradicated in the past. 55 patients with $\mathrm{Hp}$ re-infection and 95 patients not re-infected.

We interviewed the children with questionnaire about socioeconomics factors, hygiene, living conditions and their dietary habits.

Results A lower frequency of fermented dairy food, fruits and vegetable consumption was registred among children with $\mathrm{Hp}$ reinfection as compared to not been re-infected.

Among persons with Hp re-infection were noted low socio-economic markers such as croweded living conditions, a large number of siblings and unclean water.

Conclusions Might decrease the risk of Hp re-infection the use of probiotic, vitamin $\mathrm{C}$, antioxidants contained in fruit and vegetables.

Risk factors for Hp re-infection are low socioeconomics factors, hygiene and living conditions.

\section{RSV HOSPITALIZATION IN INFANTS WITH NEUROMUSCULAR DISEASE IN THE CANADIAN REGISTRY OF SYNAGIS ${ }^{\circledR}$ (CARESS) FOLLOWING PROPHYLAXIS (2005-2011)}

doi:10.1136/archdischild-2012-302724.086

'B Paes, ${ }^{2} \mathrm{~A}$ Li, ${ }^{3} \mid$ Mitchell, ${ }^{2} \mathrm{KL}$ Lanctot. 'Paediatrics, McMaster University, Hamilton; ${ }^{2}$ Medical Outcomes and Research in Economics (MORE), Sunnybrook Health Sciences Centre, Toronto, ON; ${ }^{3}$ Paediatrics (Respirology Division), University of Calgary, Calgary, $A B$, Canada

Background and Aims The Canadian Registry of Synagis $\AA$ (CARESS) tracks palivizumab use and respiratory outcomes in highrisk infants, including those with neuromuscular impairments (NMI). We compared respiratory illness (RI) and respiratory syncytial virus positive hospitalization (RSVH) rates in NMI infants versus: 1) those with other underlying medical disorders (MD) and 2) those prophylaxed for standard indications (SD).

Methods A prospective, observational registry of infants from 30 Canadian sites who received $\geq 1$ dose of palivizumab during the 2005-2011 RSV seasons. Utilization and RI events were collected monthly throughout each season.

Results 10452 infants were recruited (NMI: 118, 1.1\%; MD: 1443, 13.8\%; SD: 8891, 85.1\%). There were statistically significant group differences $(p<0.05)$ in: enrolment weight and age, gestational age, birth weight, proportions of: Caucasians, daycare attendance, smoking exposure, siblings, multiple birth, $>5$ individuals in the household, and history of atopy. NMI infants tended to have a less complex neonatal course. Compliance was similar across the three groups. The NMI group had higher RI hospitalization rates than $\mathrm{MD}$ or $\mathrm{SD}(17.8 \%$ versus $9.6 \%$ and $5.8 \%, \mathrm{p}<0.0005)$, as well as RSVH (5.62\% versus $1.98 \%$ and $1.49 \%, \mathrm{p}<0.0005)$. A Cox proportional hazard analysis showed that having NMI increased the risk of first RSVH compared to infants in the SD group (hazard ratio $=4.47$, 95\% CI 1.96-10.18, p<0.0005).

Conclusions NMI infants comprise a very high risk cohort for RI and RSV-related hospitalization and should be considered for palivizumab prophylaxis to reduce incurred morbidities as recommended by the American Academy of Pediatrics and other international advisory bodies. THE CANADIAN REGISTRY OF SYNAGIS ${ }^{\circledR}$ (CARESS) FOLLOWING PROPHYLAXIS (2005-2011)

doi:10.1136/archdischild-2012-302724.0862 